\title{
Prediction of Gas Concentration Based on the Opposite Degree Algorithm
}

\author{
Xiao-Guang Yue ${ }^{1}$ and Michael McAleer ${ }^{2-6,{ }^{*}}$ \\ ${ }^{1}$ School of Civil Engineering, Wuhan University, China \\ ${ }^{2}$ Department of Quantitative Finance, National Tsing Hua University, Taiwan \\ ${ }^{3}$ Discipline of Business Analytics, University of Sydney Business School, Australia \\ ${ }^{4}$ Econometric Institute, Erasmus School of Economics, Erasmus University Rotterdam, The Netherlands \\ ${ }^{5}$ Department of Quantitative Economics, Complutense University of Madrid, Spain \\ ${ }^{6}$ Institute of Advanced Sciences, Yokohama National University, Japan
}

\begin{abstract}
In order to study the dynamic changes in gas concentration, to reduce gas hazards, and to protect and improve mining safety, a new method is proposed to predict gas concentration, based on the opposite degree algorithm. A priori and a posteriori values, opposite degree computation, opposite space, prior matrix, and posterior matrix are 6 basic concepts of the opposite degree algorithm. Several opposite degree numerical formulae to calculate the opposite degrees between gas concentration data and gas concentration data trends can be used to predict empirical results. The opposite degree numerical computation (OD-NC) algorithm has greater accuracy than several common prediction methods, such as RBF (Radial Basis Function) and GRNN (General Regression Neural Network). The prediction mean relative errors of RBF, GRNN and OD-NC are $7.812 \%, 5.674 \%$ and $3.284 \%$, respectively. The simulation experiments show that the OD-NC algorithm is feasible and effective in practice.
\end{abstract}

Keywords: Gas concentration, opposite degree algorithm, data prediction, mining safety, numerical simulations.

\section{INTRODUCTION}

\subsection{Background and Literature Review}

Mine gases can result in hypoxia, asphyxia, or even burning and explosions, so it is an important indicator to detect gas concentration for coal mine gas safety. Gas concentration must be maintained at a reasonable level as it indicates that the coal mine is safe. In China, for example, gas concentration security issues lead to surprising that significant research has been conducted to predict gas concentration. In 1993, numerical methods were used to predict gas concentration (Fang, 1993). The gas concentration fuzzy time series forecasting model was established in 1995, and was applied to the Sichuan Furong Mining Bureau Baijiao Mine. The model to prevent gas explosion accidents was subsequently used to predict gas concentration (Zhou and Huang, 1995).

Table 1: Annual Coal Mine Gas Accidents, 2008 - 2012

\begin{tabular}{|c|c|c|c|c|c|c|}
\hline Year & $\mathbf{2 0 0 8}$ & $\mathbf{2 0 0 9}$ & $\mathbf{2 0 1 0}$ & $\mathbf{2 0 1 1}$ & $\mathbf{2 0 1 2}$ & Total Number \\
\hline \hline Accidents Times & 182 & 157 & 145 & 119 & 72 & 675 \\
\hline Number of Deaths & 778 & 755 & 623 & 553 & 459 & 3039 \\
\hline
\end{tabular}

many casualties each year, as shown in Table 1 (Zhou, 2014). For available data from 2008 to 2012, total Chinese coal mine gas accidents totalled 675 , which led to 3039 deaths.

At the international level, prediction of gas concentration is also a serious issue, so it is not

*Address of correspondence to this author at the Department of Quantitative Finance, National Tsing Hua University, Taiwan; Tel: +886-3-571-5131 ext. 62534; Fax: +886-3-562-1823; E-mail: michael.mcaleer@gmail.com

JEL: C53, C63, L71
In 2008, echoes were used to monitor coal mine gas concentration (Obst, et al. 2008), while the mixed adaptive system prediction method of time series continuous gas monitoring was presented (Sikora, et al. 2008). In 2010, forecasting methods based on the gas concentration HJM model and Monte Carlo simulation methods were analyzed (Wei, et al. 2010).

The gas concentration prediction methods based on transformed wavelet and optimized predictor was analyzed in 2011 (Wang, et al. 2011). In 2012 gas concentration and prominent detection security became 
important for the protection of mine workers and areas close to the mines, and gas wireless sensor networks (WSN) were designed, where the semiconductor gas sensor had a low power consumption and high sensitivity. Optimizing power is based on energy efficient clustering protocols, so that the goal of outburst warning can be achieved (Unnikrishna Menon, et al. 2012).

\subsection{Research Objective}

Intelligent methods in the prediction of gas emission rates and concentration are increasing, including neural networks and genetic algorithms. These methods have certain advantages and disadvantages. For example, neural networks require a large amount of data and are prone to the "less learning" and "over-learning" phenomena, leading to prediction accuracy that is not high. BP neural network will be affected by the network structure, with initial connection weights and thresholds set for training ( $\mathrm{Li}, 2006)$.

Genetic algorithms use selecting, crossover and mutation operations for computation. It has a strong global processing capacity, but local processing capacity can be weak (Deng and Liang, 2009). Therefore, in order to further improve forecast accuracy and the adaptability of the algorithm, in this paper we propose a new intelligent algorithm which can be applied to the prediction of mine gas concentration.

\section{RESEARCH METHOD AND THEORETICAL FRAMEWORK}

\subsection{Basic Concepts of the Opposite Degree Algorithm}

Gas data are dynamic, and the gas relationship with available data can be represented by the Opposite Degree (OD). The greater is the opposite degree, the greater are the differences between two associated variables. Correspondingly, the smaller is the opposite degree, the smaller are the differences between two associated variables. The opposite degrees are predominantly related with the following six concepts (Yue, et al. 2015; Yue, et al. 2016; Wang, et al. 2014; Zhou and Yue, 2015):

\section{1) A Priori Value}

A prior value refers to the data that have been used for training, and hence are obtained in advance.

\section{2) A Posteriori Value}

A posteriori analysis is used to predict the value associated with a certain set of properties.

\section{3) Opposite Degree Computation}

Opposite degree is the difference between the a priori and a posteriori values, and ranges from $(-\infty,+\infty)$.

Usually, the a priori value is $A$, the a posteriori value is $B$, denoted as $O(A, B)$, and can be obtained using formula (1), as follows:

$$
O(A, B)=\frac{B-A}{A}=\left\{\begin{array}{c}
\text { negative, for } B<A \\
0, \text { for } A=B \\
\text { positive, for } B>A
\end{array}\right.
$$

\section{4) Opposite Space}

Flat space and three-dimensional space are the most common vector spaces. The flat space is the vector space $R^{2}$, which consists of the real pairs:

$$
R^{2}=\{(x, y): x, y \in R\} .
$$

The vector space $R^{3}$ is three-dimensional space, which consists of all ordered triple real arrays:

$$
R^{3}=\{(x, y, z): x, y, z \in R\} .
$$

The concept of $R^{2}$ and $R^{3}$ can be extended to highdimensional space. Setting the length as $n$, an ordered array (vector space $R^{n}$ ) is given in equation (4):

$$
R^{n}=\left\{\left(x_{1}, x_{2}, \ldots, x_{n}\right): x_{1}, x_{2}, \ldots, x_{n} \in R\right\}
$$

where $j \in\{1,2, \ldots, n\}$.

In the multi-dimensional space, the calculation of the opposite degree is changed to vector calculation. The a priori value is changed to the a priori vector, $A=\left(A_{1}, A_{2}, \ldots, A_{m}\right)$, while the a posteriori value is changed to the a posteriori vector, $B=\left(B_{1}, B_{2}, \ldots, B_{m}\right)$. The calculations are shown in equations (5) and (6), respectively:

$$
\begin{aligned}
& O(A, B)=\left\{O_{1}\left(A_{1}, B_{1}\right), O_{2}\left(A_{2}, B_{2}\right), \ldots, O_{m}\left(A_{m}, B_{m}\right)\right\} \\
& O(A, B)=\left(\begin{array}{c}
O_{1}\left(A_{1}, B_{1}\right) \\
O_{2}\left(A_{2}, B_{2}\right) \\
\ldots \\
O_{m}\left(A_{m}, B_{m}\right)
\end{array}\right)
\end{aligned}
$$

\section{5) A Priori Matrix}

The a priori matrix is a matrix (data set) that is used to train the prediction method. The a priori matrices are 
obtained in advance of the numerical matrix, consisting of the a priori numerical composition, $a_{i j}\left(a_{i j} \in A_{m \times n}\right.$, $1 \leq i \leq m, \quad 1 \leq j \leq n)$. They include the $n$ column properties, with each column having $m$ row data, and each row of data corresponding to the a priori values, $r_{i}\left(r_{i} \in R, 1 \leq i \leq m\right)$.

The given prior matrix, $A_{m \times n}$, is shown in equation (7):

$$
A_{m \times n}=\left[\begin{array}{cccc}
a_{11} & a_{12} & \ldots & a_{1 n} \\
a_{21} & a_{22} & \ldots & a_{2 n} \\
\ldots & \ldots & \ldots & \ldots \\
a_{m 1} & a_{m 2} & \ldots & a_{m n}
\end{array}\right]
$$

where its a priori numerical column vector is $R$, namely:

$$
R=\left[\begin{array}{c}
r_{1} \\
r_{2} \\
\cdots \\
r_{m}
\end{array}\right]
$$

\section{6) A Posteriori Matrix}

The a posteriori matrix refers to a matrix (data set) that is used for predictive analysis. The a posteriori matrix is composed of the value of the a posteriori values, $b_{k j}\left(b_{k j} \in B_{p \times n}, 1 \leq k \leq p, 1 \leq j \leq n\right)$, including $n$ column properties, with each column property having $p$ rows of data, and each row corresponding to the a posterior value, $s_{k} \quad\left(s_{k} \in S, 1 \leq k \leq p\right)$. Opposite degree calculations require the a posteriori predicted values.

The given a posteriori matrix, $B_{p \times n}$, is shown in equation (9):

$$
B_{p \times n}=\left[\begin{array}{cccc}
b_{11} & b_{12} & \ldots & b_{1 n} \\
b_{21} & b_{22} & \ldots & b_{2 n} \\
\ldots & \ldots & \ldots & \ldots \\
b_{p 1} & b_{p 2} & \ldots & b_{p n}
\end{array}\right]
$$

The opposite degree algorithm can be used to predict the value of the a posteriori matrix, $B_{p \times n}$, corresponding to the column vector, $S$ :

$$
S=\left[\begin{array}{c}
s_{1} \\
s_{2} \\
\cdots \\
s_{m}
\end{array}\right]
$$

\subsection{Opposite Degree Algorithm - Numerical Computation}

As there can be many non-linear relationships, how to calculate the distance between these values is very important. We consider using the method of numerical calculation of the opposite degree algorithm. The smaller is the opposite degree between two gas emission quantities, the closer they will be, and when two gas emission opposite degrees are greater, the further apart will the difference be between them. Similarly, the smaller are the opposite degree related indicators between two sets of gas emission data, the closer they will be, and the greater are the opposite degree related indicators between two sets of gas emission data, the greater will be the difference.

Due to the opposite degree vector being constructed by opposite degree components, the opposite degree space is constituted by opposite degree vectors. Therefore, the calculation of the opposite degrees is clearly closed, resulting in the collection of the operation as the opposite degree space. The main steps of the opposite degree numerical computation algorithm can be summarized in the following four steps:

1) Establish populations of neural networks, and compute the basic characteristics of the a priori values. Calculating the value of the basic characteristics of the a priori initial opposite degree of the individual data from the population correlation network will facilitate calculation of the weights.

2) Assess the fitness by calculating the weights, which are used to give the "individual data items I indicators" relative importance, and then select the value of the a posteriori values.

3) Select high fitness data. The opposite degree algorithm performs the calculations, and the comparisons are based on the weights and various parameters.

4) According to the parameters calculated, the a posteriori values are predicted.

As a result, the opposite degree numerical computation (OD-NC) method can be used based on matrix operations. The OD-NC steps are given as follows:

1) Calculate the opposite degree matrix of the training sample. The opposite degree matrix, $O_{i}$ 
$(1 \leq i \leq m)$, is the opposite degree between the $i$ th row of $A_{m \times n} R$ and the $m$ th row of $A_{m \times n} R$, where $A_{m \times n} R$ is composed of matrix $A_{m \times n}$ and vector $R$, with $m$ rows and $n+1$ columns. The calculation of the matrix of opposite degrees is given in equations (11) and (12):

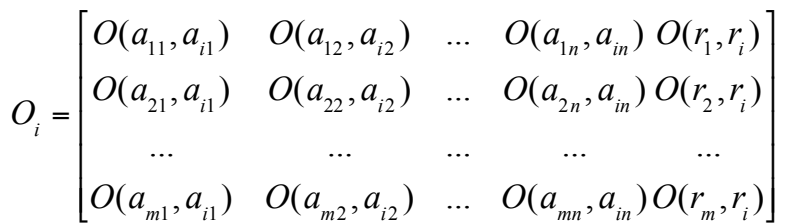

$O_{i}=\left[\begin{array}{cccc}\frac{a_{i 1}-a_{11}}{a_{11}} & \frac{a_{i 2}-a_{12}}{a_{12}} & \ldots & \frac{a_{i n}-a_{1 n}}{a_{1 n}} \frac{r_{i}-r_{1}}{r_{1}} \\ \frac{a_{i 1}-a_{21}}{a_{21}} & \frac{a_{i 2}-a_{22}}{a_{22}} & \ldots & \frac{a_{i n}-a_{2 n}}{a_{2 n}} \frac{r_{i}-r_{2}}{r_{2}} \\ \ldots & \ldots & \ldots & \ldots \\ \frac{a_{i 1}-a_{m 1}}{a_{m 1}} & \frac{a_{i 2}-a_{m 2}}{a_{m 2}} & \ldots & \frac{a_{i n}-a_{m n}}{a_{m n}} \frac{r_{i}-r_{m}}{r_{m}}\end{array}\right]$

2) Calculate weights, where $\omega_{j}$ represents the weight of each data $\left(a_{i j}\right)$, and is based on the following steps:

(1) Remove all the 0 rows from matrix $O_{i}$, which gives $O_{i}^{\prime}$, as all the 0 rows cannot be used to compute the weights. There are $i$ matrices, which can be composed of matrix $O^{\prime}$, as shown in equation (13):

$O^{\prime}=\left[\begin{array}{c}O_{1}^{\prime} \\ O_{2}^{\prime} \\ \ldots \\ O_{m}^{\prime}\end{array}\right]=\left[\begin{array}{ccccc}O\left(a_{21}, a_{11}\right) & O\left(a_{22}, a_{12}\right) & \ldots & O\left(a_{2 n}, a_{1 n}\right) & O\left(r_{2}, r_{1}\right) \\ O\left(a_{31}, a_{11}\right) & O\left(a_{32}, a_{12}\right) & \ldots & O\left(a_{3 n}, a_{1 n}\right) & O\left(r_{3}, r_{1}\right) \\ \ldots & \ldots & \ldots & \ldots & \ldots \\ O\left(a_{m 1}, a_{11}\right) & O\left(a_{m 2}, a_{12}\right) & \ldots & O\left(a_{m n}, a_{1 n}\right) & O\left(r_{m}, r_{1}\right) \\ O\left(a_{11}, a_{21}\right) & O\left(a_{12}, a_{22}\right) & \ldots & O\left(a_{1 n}, a_{2 n}\right) & O\left(r_{1}, r_{2}\right) \\ O\left(a_{31}, a_{21}\right) & O\left(a_{32}, a_{22}\right) & \ldots & O\left(a_{3 n}, a_{2 n}\right) & O\left(r_{3}, r_{2}\right) \\ \ldots & \ldots & \ldots & \ldots & \ldots \\ O\left(a_{m 1}, a_{21}\right) & O\left(a_{m 2}, a_{22}\right) & \ldots & O\left(a_{m n}, a_{2 n}\right) & O\left(r_{m}, r_{2}\right) \\ \ldots & \ldots & \ldots & \ldots & \ldots \\ O\left(a_{11}, a_{m 1}\right) & O\left(a_{12}, a_{m 2}\right) & \ldots & O\left(a_{1 n}, a_{m n}\right) & O\left(r_{1}, r_{m}\right) \\ O\left(a_{21}, a_{m 1}\right) & O\left(a_{22}, a_{m 2}\right) & \ldots & O\left(a_{2 n}, a_{m n}\right) & O\left(r_{2}, r_{m}\right) \\ \ldots & \ldots & \ldots & \ldots & \ldots \\ O\left(a_{m-1,1}, a_{m 1}\right) & O\left(a_{m-1,2}, a_{m 2}\right) & \ldots & O\left(a_{m-1, n}, a_{m n}\right) & O\left(r_{m-1}, r_{m}\right)\end{array}\right]$

(2) The last column is a reference value for prediction, namely $O^{\prime \prime}$. The calculation of the matrix of the weights is given in equation (14):
$O^{n}=\left[\begin{array}{c}O_{1}^{\prime \prime} \\ O_{2}^{\prime \prime} \\ \cdots \\ O_{m}^{\prime}\end{array}\right]=$

$\left[\begin{array}{llll}O\left(O\left(r_{2}, r_{1}\right), O\left(a_{21}, a_{11}\right)\right) & O\left(O\left(r_{2}, r_{1}\right), O\left(a_{22}, a_{12}\right)\right) & \ldots & O\left(O\left(r_{2}, r_{1}\right), O\left(a_{2 n}, a_{1 n}\right)\right) \\ O\left(O\left(r_{3}, r_{1}\right), O\left(a_{31}, a_{11}\right)\right) & O\left(O\left(r_{3}, r_{1}\right), O\left(a_{32}, a_{12}\right)\right) & \ldots & O\left(O\left(r_{3}, r_{1}\right), O\left(a_{3 n}, a_{1 n}\right)\right) \\ \ldots & \ldots & \ldots & \ldots \\ O\left(O\left(r_{m}, r_{1}\right), O\left(a_{m 1}, a_{11}\right)\right) & O\left(O\left(r_{m}, r_{1}\right), O\left(a_{m 2}, a_{12}\right)\right) & \ldots & O\left(O\left(r_{m}, r_{1}\right), O\left(a_{m n}, a_{1 n}\right)\right) \\ O\left(O\left(r_{1}, r_{2}\right), O\left(a_{11}, a_{21}\right)\right) & O\left(O\left(r_{1}, r_{2}\right), O\left(a_{12}, a_{22}\right)\right) & \ldots & O\left(O\left(r_{1}, r_{2}\right), O\left(a_{1 n}, a_{2 n}\right)\right) \\ O\left(O\left(r_{3}, r_{2}\right), O\left(a_{31}, a_{21}\right)\right) & O\left(O\left(r_{3}, r_{2}\right), O\left(a_{32}, a_{22}\right)\right) & \ldots & O\left(O\left(r_{3}, r_{2}\right), O\left(a_{3 n}, a_{2 n}\right)\right) \\ \ldots & \ldots & \ldots & \ldots \\ O\left(O\left(r_{m}, r_{2}\right), O\left(a_{m 1}, a_{21}\right)\right) & O\left(O\left(r_{m}, r_{2}\right), O\left(a_{m 2}, a_{22}\right)\right) & \ldots & O\left(O\left(r_{m}, r_{2}\right), O\left(a_{m n}, a_{2 n}\right)\right) \\ \ldots & \ldots & \ldots & \ldots \\ O\left(O\left(r_{1}, r_{m}\right), O\left(a_{11}, a_{m 1}\right)\right) & O\left(O\left(r_{1}, r_{m}\right), O\left(a_{12}, a_{m 2}\right)\right) & \ldots & O\left(O\left(r_{1}, r_{m}\right), O\left(a_{1 n}, a_{m n}\right)\right) \\ O\left(O\left(r_{2}, r_{m}\right), O\left(a_{21}, a_{m 1}\right)\right) & O\left(O\left(r_{2}, r_{m}\right), O\left(a_{22}, a_{m 2}\right)\right) & \ldots & O\left(O\left(r_{2}, r_{m}\right), O\left(a_{2 n}, a_{m n}\right)\right) \\ \ldots & \ldots & \ldots & \ldots \\ O\left(O\left(r_{m-1}, r_{m}\right), O\left(a_{m-1,1}, a_{m 1}\right)\right) & O\left(O\left(r_{m-1}, r_{m}\right), O\left(a_{m-1,2}, a_{m 2}\right)\right) & \ldots & O\left(O\left(r_{m-1}, r_{m}\right), O\left(a_{m-1, n}, a_{m n}\right)\right)\end{array}\right]$

(3) Calculate absolute values, where the weights denote the important levels:

$\left|O^{\prime \prime}\right|=\left[\begin{array}{c}\left|O_{1}^{\prime \prime}\right| \\ \left|O_{2}^{\prime \prime}\right| \\ \cdots \\ \left|O_{m}^{\prime \prime}\right|\end{array}\right]$

(4) Calculate the mean value of each column. Prior to any calculations, part of the column may contain " $E$ ", in which case the results of the "E" are deleted from the data, and calculate the mean value, with $\overline{\omega_{i}}$ taken as the mean value for each column. The default value of the " $E$ " is 0 . The calculations are given in equation (16):

$$
\begin{gathered}
\sum\left(\left|O\left(O\left(r_{2}, r_{1}\right), O\left(a_{2 i}, a_{1 i}\right)\right)\right|+\mid O\left(O\left(r_{3}, r_{1}\right),\right.\right. \\
\bar{\omega}_{i}=\frac{\left.\left.O\left(a_{3 i}, a_{1 i}\right)\right)|+\ldots+| O\left(O\left(r_{m-1}, r_{m}\right), O\left(a_{m-1, i}, a_{m i}\right)\right) \mid\right)}{m \cdot(m-1)}
\end{gathered}
$$

(5) Calculate the weight. First, calculate the reciprocal of the mean value; second, obtain the sum; and finally, divide the total weight by the sum. The weight for each column is $\omega_{j}$, and the calculation is given in equation (17):

$\omega_{i}=\frac{\frac{1}{\overline{\omega_{i}}}}{\frac{1}{\omega_{1}}+\frac{1}{\omega_{2}}+\ldots+\frac{1}{\omega_{n}}}$

3) Calculate the prediction sample opposite degree matrix. $\hat{O}_{k}$ is the opposite degree matrix based on the opposite degree calculation of row $k$ 
$(1 \leq k \leq p)$ of $B_{p \times n}$ and row $m$ of $A_{m \times n}$, as given below:

$$
\begin{aligned}
& \hat{O}_{k}=\left[\begin{array}{cccc}
O\left(a_{11}, b_{k 1}\right) & O\left(a_{12}, b_{k 2}\right) & \ldots & O\left(a_{1 n}, b_{k n}\right) \\
O\left(a_{21}, b_{k 1}\right) & O\left(a_{22}, b_{k 2}\right) & \ldots & O\left(a_{2 n}, b_{k n}\right) \\
\ldots & \ldots & \ldots & \ldots \\
O\left(a_{m 1}, b_{k 1}\right) & O\left(a_{m 2}, b_{k 2}\right) & \ldots & O\left(a_{m n}, b_{k n}\right)
\end{array}\right] \\
& =\left[\begin{array}{cccc}
\frac{b_{k 1}-a_{11}}{a_{11}} & \frac{b_{k 2}-a_{12}}{a_{12}} & \ldots & \frac{b_{k n}-a_{1 n}}{a_{1 n}} \\
\frac{b_{k 1}-a_{21}}{a_{21}} & \frac{b_{k 2}-a_{22}}{a_{22}} & \ldots & \frac{b_{k n}-a_{2 n}}{a_{2 n}} \\
\ldots & \ldots & \ldots & \ldots \\
\frac{b_{k 1}-a_{m 1}}{a_{m 1}} & \frac{b_{k 2}-a_{m 2}}{a_{m 2}} & \ldots & \frac{b_{k n}-a_{m n}}{a_{m n}}
\end{array}\right]
\end{aligned}
$$

4) Calculate the test sample's mean opposite degree and weighted opposite degree. The calculation methods of the mean opposite degree, $\xi_{k j}(1 \leq i \leq m, 1 \leq k \leq p, 1 \leq j \leq m)$, and the weighted opposite degree, $\hat{\xi}_{k j}(1 \leq i \leq m, 1 \leq k \leq p, 1 \leq j \leq m)$, are given in equations (19) and (20), respectively:

$\xi_{k j}=\frac{\sum\left(O\left(a_{i 1}, b_{k}\right)+O\left(a_{i 2}, b_{k 2}\right)+\ldots+O\left(a_{i n}, b_{k n}\right)\right)}{n}$

$$
\hat{\xi}_{k j}=\sum\left(O\left(a_{i 1}, b_{k 1}\right) \cdot \omega_{1}+O\left(a_{i 2}, b_{k 2}\right) \cdot \omega_{2}+\ldots+O\left(a_{i n}, b_{k n}\right) \cdot \omega_{n}\right) .
$$

5) Calculate alternative data row. According to equation (21), select the rows that contain $\beta_{k l}(1 \leq k \leq p, 1 \leq l \leq n)$ as the alternative row:

$\beta_{k l}=\min \left(\left|O\left(a_{1 n}, b_{k n}\right)\right|,\left|O\left(a_{2 n}, b_{k n}\right)\right|, \ldots,\left|O\left(a_{m n}, b_{k n}\right)\right|\right)$.

6) Find the basis data row. Select the row with minimum value $\left(\min \left(\xi_{k j}\right)\right)$, and record its real value as $s_{k}$.

7) Calculate the result matrix based on equation (22):

$\hat{s_{k}}=s_{k} \times\left(1+\hat{\xi_{k j}}\right)$,

where the output is the result matrix:

$$
\hat{S}=\left[\begin{array}{c}
\wedge \\
s_{1} \\
\hat{s_{2}} \\
\cdots \\
\hat{s}_{p}
\end{array}\right]
$$

\section{GAS CONCENTRATION ANALYSIS AND PREDICTION BASED ON OD-NC}

\subsection{Data Sources}

We select several commonly used variables in the analysis of gas concentration, namely wind speed $(\mathrm{m} / \mathrm{s})$, temperature $\left({ }^{\circ} \mathrm{C}\right)$, negative pressure $(\mathrm{kpa}), \mathrm{CO}$ concentration $(p p m)$, and 3 minutes for the interval form time series, as shown in Table 2 (Zeng, 2011).

The following are used to identify the variables, where $G_{1}, F_{1}, F_{2}, F_{3}, F_{4}$ are wind speed $(\mathrm{m} / \mathrm{s})$, temperature $\left({ }^{\circ} \mathrm{C}\right)$, negative pressure $(\mathrm{kpa})$, and $\mathrm{CO}$ concentration $(p p m)$, respectively.

The time series is not included in the calculations, and the final gas concentration data are shown in Table 3. In the comparisons, we select the top 22 groups as training samples, and the last 8 groups as test samples.

\subsection{Gas Concentration Prediction Based on OD-NC}

The calculation procedure is based on the OD-NC algorithm. The prediction results are given in equation (24):

$\hat{S}=\left[\begin{array}{l}0.9671 \\ 0.9096 \\ 0.8966 \\ 0.9449 \\ 0.9449 \\ 0.9628 \\ 0.9464 \\ 0.9265\end{array}\right]$

\subsection{Analysis and Findings}

In order to analyze the accuracy of the OD-NC algorithm, we provide a comparative analysis of the RBF, GRNN and OD-NC algorithms. The RBF (Radial Basis Function) is a radial basis function neural network, and the GRNN (General Regression Neural Network) algorithm is a generalized regression neural network.

These three algorithms are designed for processing the data in Table 3. For purposes of comparison with the real values, the three methods are used to predict the real values in Table 4 and Figure 1, to predict the relative errors of the results in Table $\mathbf{5}$, and to predict the mean relative errors of the results in Table 6. 
Table 2: Gas Concentration Data

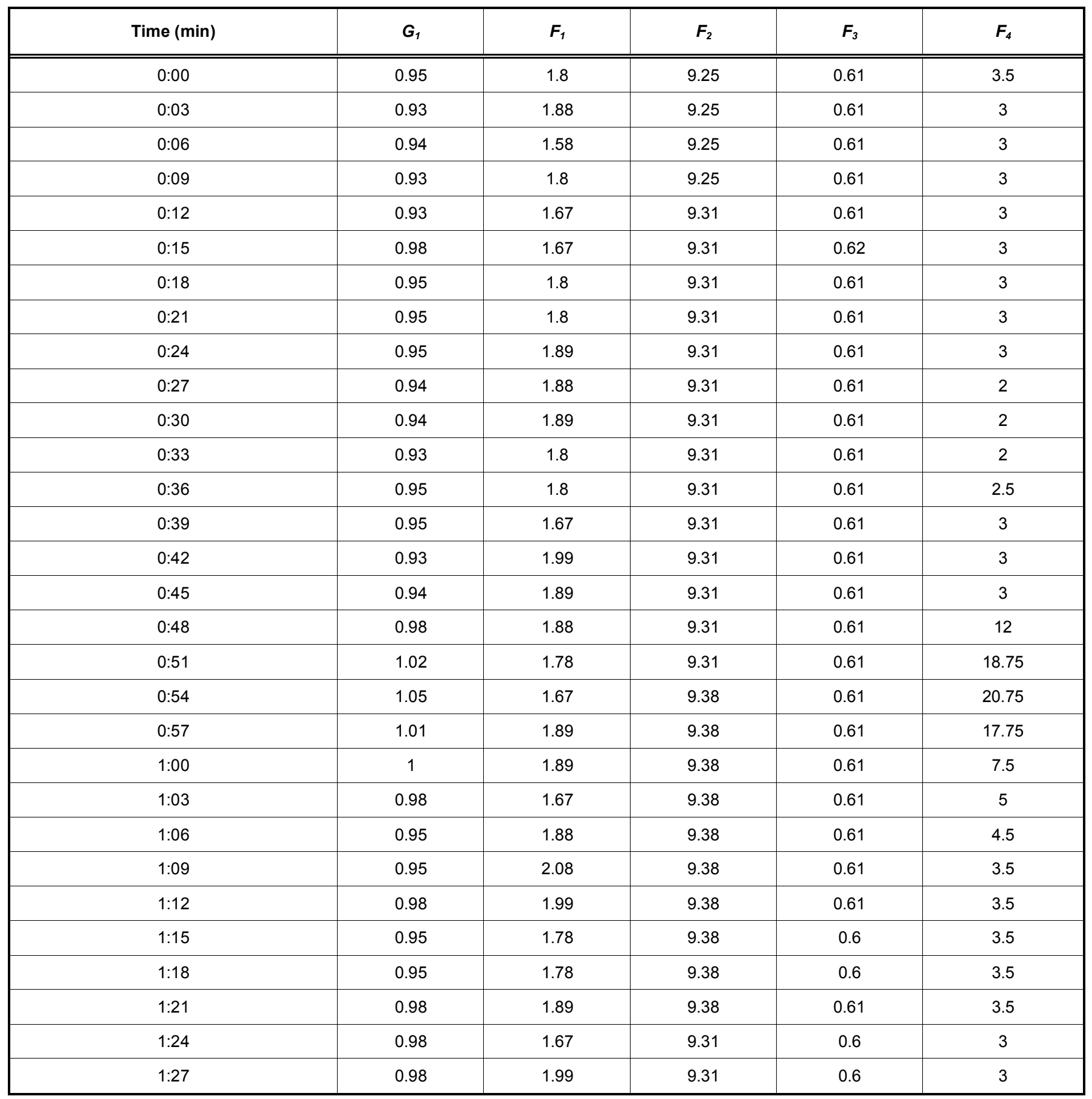

From the above tables, the relative error and mean relative error can be compared. The OD-NC algorithm is superior to the RBF and GRNN algorithms. From Figure 1, it is clear that the prediction results from the different methods show that the real value is close to the OD-NC prediction results. In Table 6, the OD-NC relative error control in less than $4 \%$. Therefore, the OD-NC algorithm is a feasible and effective method for predicting gas concentration.

\section{CONCLUSION AND DISCUSSION}

The paper introduced the concept of opposites, constructed the opposite degree algorithm, and discussed the OD-NC algorithm, as follows: (1) the opposite degree algorithm was obtained by cyclic matrix and data, and expressed the relationship of the training data; (2) calculated the effect of data and matrix of the predicted value, obtained the degree of 
Table 3: Final Gas Concentration Data

\begin{tabular}{|c|c|c|c|c|c|}
\hline No. & $F_{1}$ & $F_{2}$ & $F_{3}$ & $F_{4}$ & $G_{1}$ \\
\hline 1 & 1.8 & 9.25 & 0.61 & 3.5 & 0.95 \\
\hline 3 & 1.58 & 9.25 & 0.61 & 3 & 0.94 \\
\hline 4 & 1.8 & 9.25 & 0.61 & 3 & 0.93 \\
\hline 7 & 1.8 & 9.31 & 0.61 & 3 & 0.95 \\
\hline 8 & 1.8 & 9.31 & 0.61 & 3 & 0.95 \\
\hline 9 & 1.89 & 9.31 & 0.61 & 3 & 0.95 \\
\hline 10 & 1.88 & 9.31 & 0.61 & 2 & 0.94 \\
\hline 14 & 1.67 & 9.31 & 0.61 & 3 & 0.95 \\
\hline 15 & 1.99 & 9.31 & 0.61 & 3 & 0.93 \\
\hline 16 & 1.89 & 9.31 & 0.61 & 3 & 0.94 \\
\hline 17 & 1.88 & 9.31 & 0.61 & 12 & 0.98 \\
\hline 18 & 1.78 & 9.31 & 0.61 & 18.75 & 1.02 \\
\hline 19 & 1.67 & 9.38 & 0.61 & 20.75 & 1.05 \\
\hline 20 & 1.89 & 9.38 & 0.61 & 17.75 & 1.01 \\
\hline 21 & 1.89 & 9.38 & 0.61 & 7.5 & 1 \\
\hline 28 & 1.89 & 9.38 & 0.61 & 3.5 & 0.98 \\
\hline 29 & 1.67 & 9.31 & 0.6 & 3 & 0.98 \\
\hline 30 & 1.99 & 9.31 & 0.6 & 3 & 0.98 \\
\hline
\end{tabular}

Table 4: Prediction Results

\begin{tabular}{|c|c|c|c|c|}
\hline \multirow{2}{*}{ No. } & \multirow{2}{*}{ Real Value (\%) } & \multicolumn{3}{|c|}{ Prediction Value (\%) } \\
\cline { 2 - 5 } & & OD-NC & \multicolumn{2}{|c|}{ RBF } \\
\hline \hline 1 & 0.95 & 0.9671 & 0.7791 & 0.9571 \\
\hline 2 & 0.95 & 0.9096 & 0.8919 & 0.8089 \\
\hline 3 & 0.98 & 0.8966 & 0.9274 & 0.9528 \\
\hline 4 & 0.95 & 0.9449 & 0.9274 & 0.9528 \\
\hline 5 & 0.95 & 0.9449 & 0.9229 & 0.9540 \\
\hline 6 & 0.98 & 0.9628 & 0.8998 & 0.8994 \\
\hline 7 & 0.98 & 0.9464 & 0.9117 & 0.8910 \\
\hline
\end{tabular}


Table 5: Relative Errors of the Three Methods

\begin{tabular}{|c|l|l|c|}
\hline \multirow{2}{*}{ No. } & \multicolumn{3}{|c|}{ Relative Error (\%) } \\
\cline { 2 - 4 } & OD-NC & RBF & 0.0078 \\
\hline \hline 1 & 0.0180 & 0.1799 & 0.1485 \\
\hline 2 & 0.0425 & 0.0978 & 0.0921 \\
\hline 3 & 0.0851 & 0.0899 & 0.0029 \\
\hline 4 & 0.0054 & 0.0238 & 0.0029 \\
\hline 5 & 0.0054 & 0.0238 & 0.0265 \\
\hline 6 & 0.0176 & 0.0583 & 0.0822 \\
\hline 8 & 0.0343 & 0.0818 & 0.0908 \\
\hline
\end{tabular}

Table 6: Mean Relative Errors of the Three Methods

\begin{tabular}{|c|c|c|}
\hline Method & OD-NC & RBF \\
\hline \hline Mean Relative Error (\%) & 3.2837 & 7.8120 \\
\hline
\end{tabular}

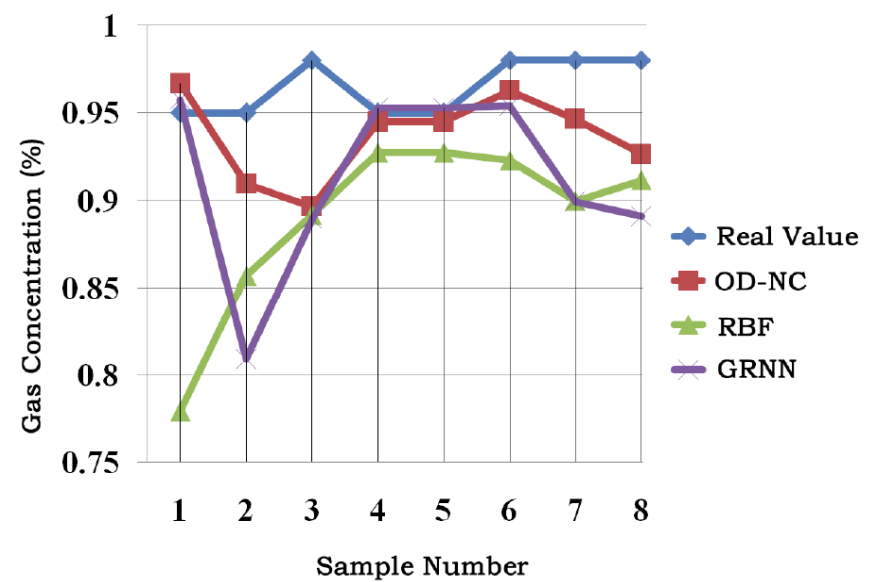

Figure 1: Prediction results.

opposition, and then analyzed the influencing factors of weights; (3) according to the weight values, and testing data of opposite degree mean and the weighted opposite degree, determined the reference for calculation; and (4) through the opposite degree algorithm, it is possible to predict numerical data.

The prediction results showed that: (1) the OD-NC algorithm was superior to the competing RBF and GRNN algorithms; and (2) the mean error of the ODNC algorithm was controlled within $4 \%$, which was lower than for the other methods. Therefore, the OD$\mathrm{NC}$ algorithm is feasible and effective in the prediction and analysis of gas concentration in practice.

Gas concentration is an important test data of the mine. A dangerous gas situation can cause a large number of casualties and property losses, and affect economic efficiency. By analyzing and predicting gas concentration, the future security of the mines can be evaluated. In order to reduce casualties, material and financial losses, we can use the new algorithm to predict gas concentration.

Future research will continue to improve the accuracy of the algorithm, and apply the algorithm to additional practical projects.

\section{CONFLICT OF INTEREST}

The authors confirm that the article content has no conflict of interest.

\section{SUPPORT}

For financial support, the second author wishes to acknowledge the Australian Research Council and the National Science Council, Ministry of Science and Technology (MOST), Taiwan.

\section{REFERENCES}

Deng, Y.C., X.M. Liang (2009), Accelerating convergency genetic algorithm of improved operator, Modern Electronics Technique, 2, 139-141.

Fang, H.G. (1993), Estimation of gas emission time and prediction of gas concentration in the stop wind area, Coal Mine Safety, 9, 30-31.

Li, F.L. (2006), Study on the prediction method of neural network based on financial securities, Master Thesis, Qingdao University. 
Obst, O., X.R. Wang, M. Prokopenko (2008), Using echo state networks for anomaly detection in underground coal mines, Proceedings of 2008 International Conference on Information Processing in Sensor Networks, St. Louis, USA, pp. 219-229. https://doi.org/10.1109/ipsn.2008.35

Sikora, M., Z. Krzystanek, B. Bojko, et al. (2008), Hybrid adaptative system of gas concentration prediction in hard-coal mines, Proceedings of 19th International Conference on Systems Engineering, Las Vegas, USA, pp. 159-164. https://doi.org/10.1109/icseng.2008.32

Unnikrishna Menon, K.A., M. Deepa, H. Thirugnanam (2012), Energy efficient clustering protocol for early warning system for miner's safety in coal mines, Proceedings of 21st Annual Wireless and Optical Communications Conference, Kaohsiung, Taiwan, pp. 99-104.

Wang, X.C., X.-G. Yue, M. Ranjbar, S.K. Sanjar, M.V. Cañiv (2014), Opposite degree algorithm and its application in engineering data processing, Computer Modelling and New Technologies, 18(11), 482-485.

Wang, X.O., J. Liu, J.J. Lu (2011), Gas concentration forecasting approach based on wavelet transform and optimized predictor, Journal of Basic Science and Engineering, 19(3), 499-508.

Wei, X., R. Wang, J.T. Wu (2010), The research of gas concentration prediction model based on Monte Carlo method and HJM, Industry and Mine Automation, 5, 37-40.
Yue, X.-G., G. Zhang, Q. Wu, F. Li, X.F. Chen, G.F. Ren, M. Li (2015), Wearing prediction of stellite alloys based on opposite degree algorithm, Rare Metals, 34(2), 125-132. https://doi.org/10.1007/s12598-014-0430-0

Yue, X.-G., S.K. Boddhu, Y. Lu, F.Y. Xiao, T. Memonen and M.V. Cañiv (2016), Gas outburst prediction based on OD algorithm, Recent Patents on Computer Science, 9(1), 25-39. https://doi.org/10.2174/2213275908666150218194450

Zeng, Q.Q. (2011), Prediction of coal mine gas concentration based on quotient space, Master Thesis, Taiyuan University of Technology, China.

Zhou, C.C., Y.P. Huang (1995), All state prediction of gas concentration in working environment, Journal of Xi'an Mining Institute, 4, 315-319.

Zhou, S., X.-G. Yue (2015), Soft soil foundation settlement prediction and economic cost management analysis based on new algorithm, Proceedings of ICEMCS, Shenyang, China, 17, pp. 395-399. https://doi.org/10.2991/emcs-15.2015.81

Zhou, X.Q. (2014), Analysis of emergency management importance based on mine gas explosion accidents, Coal Mine Science and Technology, 42(1), 40-43.

https://doi.org/10.1016/j.surfcoat.2013.12.010

DOI: https://doi.org/10.6000/1929-7092.2017.06.13

(c) 2017 Yue and McAleer; Licensee Lifescience Global.

This is an open access article licensed under the terms of the Creative Commons Attribution Non-Commercial License (http://creativecommons.org/licenses/by-nc/3.0/) which permits unrestricted, non-commercial use, distribution and reproduction in any medium, provided the work is properly cited. 